\title{
AN ADAPTIVE NETWORK CONTROL SCHEME FOR REGION-BASED HYBRID CODING ALGORITHM*
}

\author{
Hsu-Tung Chen, Liang-Gee Chen, Sheng-Chieh Huang, Tsung-Han Tsai, Hao-Chieh Chang \\ Department of Electrical Engineering, National Taiwan University, \\ No. 1, Roosevelt Road, Sec. 4, Taipei, Taiwan, R.O.C.
}

\begin{abstract}
Based on the packet loss rate and the frame loss rate experiment in the practical Internet environment, a novel adaptive network control scheme is proposed for region-based hybrid coding algorithm. In comparison with the source/channel coding [1], this adaptive network control scheme is presented to reduce 50\% frame loss rate during the Internet weak day use condition and $58 \%$ frame loss rate during the Internet peak day use condition.

\section{INTRODUCTION}

In the video conference system, the compressed multimedia data is transmitted to receiver by network. The network need to guarantee the real time transmission, low delay, and low packet loss rate. In the past researches, there are the source/channel coding [1] and automatic repeat request ( $A R Q)$ [2] mechanism to solve the packet loss problem. In this paper, a novel adaptive network control scheme is applied to the region-based hybrid coding algorithm [3] for solving the packet loss problem of the multimedia compressed data.
\end{abstract}

\section{REGION-BASED HYBRID CODING ALGORITHM}

There are two important properties in the video conference system. In first, camera is fixed in video conference application. Secondly, a partocipant appears in front of an unchanged background, and moves almost slowly. The information of the background can be removed for reducing the computation loading and the network congestion.

${ }^{*}$ This work was supported by National Science Council under Grant NSC 87-2215-E-002-028.
The following example explains the region-based hybrid coding algorithm. A circle object moves in consecutive frames from lower left to upper right as shown in Fig. 1 (a) and Fig 1 (b). The difference of two frames is shown in Fig. 1 (c). It is obvious that the changed region is the two circle objects. It is only necessary to encode the two objects.

The region-based hybrid coding algorithm is shown in Fig. 2. In the coding algorithm, the $I$ frame is Intra_Frame(n) and the $P$ frame is Frame(n) - Predict_Frame(n-I) as shown in Fig. 3. The $I$ frame coding algorithm and $P$ frame coding algorithm are described in the following:

\subsection{I frame Coding Algorithm}

The $I$ frame is encoded as still image. The following is the $I$ frame coding algorithm:

Step I: I frame is divided into many $8 \times 8$ blocks.

Step 2: For each block in I frame, do the following processing:

$$
\text { / do_8x8_DCT; }
$$

$$
\text { quantization; }
$$

run length coding;

$V L C$;

add to transmission buffer container; )

\subsection{P frame Coding Algorithm}

Step 1: P frame is divided into many $8 \times 8$ blocks.

Step 2: Calculate MAE (Mean Absolute Error) for per block.

Step 3: For each block in P frame, do the following processing:

i if $(M A E>$ threshold $)$

( do_8x8_DCT; 
quantization;

run length coding;

$V L C$

add to transmission buffer container;/]

The MAE (Mean Absolute Error) and threshold are used to identify the changed blocks in $P$ frame.

\section{ADAPTIVE NETWORK CONTROL SCHEME}

The design of the adaptive network control scheme is based on the packet loss rate of the UDP protocol experiment and the frame loss simulation of the region-based coding algorithm on Internet. The two experiments are described in the following:

\subsection{The Loss Rate of the UDP Protocol Experiment:}

This experiment is performed at peak use of network and heavy load of cicsun06 (load=1.2) as shown in Fig.4. The TD is time division (ms) between the two successive packet transmissions. There are two results shown in this figure. In first, the larger packet size will have higher probability of packet loss at the same time division. Secondly, the longer time division will have lower packet loss rate at the same packet size.

\subsection{The Frame Loss Simulation of the Region- based Coding Algorithm}

Without retransmission of the lost frame, the Fig. 5 represents that the packet arrival process is highly correlated with the successive packet drops. When there are more than three successive frames loss, the $P S N R$ value of the frame will decline quickly and video quality will be poor.

\subsection{Adaptive Network Control Scheme Using First Order Markov Model}

According the above two experiments result, a novel adaptive network control scheme is proposed for the region-based hybrid coding algorithm. In this scheme, the successive packet loss condition can be detected and the frame loss rate can controlled with the adjustment of the frame transmission threshold.
The packet loss condition can be detected with the transmission status between the transmitter and the receiver. After receiver gets a video packet, it sends a grant to transmitter immediately. So, the packet loss condition can be counted and the first order Markov model is used to simulate the behavior of the network as shown in Fig. 6. In this model, there are two states and four parameters described as following:

$P S$ : the state of a successful packet.

$P L$ : the state of a loss packet.

PSS: the probability of a successful packet following a successful packet.

$P S L$ : the probability of a successful packet following a loss packet.

PLS: the probability of a loss packet following a successful packet.

PLL: the probability of a loss packet following a loss packet.

The two equations can be got as following:

$$
\begin{aligned}
& P S S+P S L=1 \\
& P L L+P L S=1
\end{aligned}
$$

Based on the above model, a novel network control scheme is designed to adapt the video transmission. This scheme degrades or upgrades the server transmission rate depending on feedback message from receiver on the control channel.

When the $P S L$ and the $P L L$ are small, it is hard to become the $P L$ status from the $P S$ status, but easy to become the $P S$ status from the $P L$ status. The two conditions ( $P S L>c p s l)$ or $(P L L>c p l l)$ are used as criteria. The $c p s l$ and $c p l l$ criteria parameters are the probability of the packet loss and used to detect the satus of the packet loss. The cpsl and cpll criteria parameters are adjusted according the necessity of the users.

If the packet loss rare is high, the threshold of the region-based hybrid coding algorithm is increased to reduce the data rate of the 
changed blocks in the $\mathrm{P}$ frame. When network is in weak use, the threshold is decreased to get high video quality. The adaptive network control scheme is described as following:

if (( PSL > cpsl ) or ( PLL > cpll))

I if (threshold < 800)

$$
\text { threshold }+=50 \text {; }
$$

else

( if (framerate $>1$ )

framerate $=$ frame $-1 ;\}$

else

$\{\quad$ if (threshold $>100$ )

$$
\text { threshold }=50 \text {; }
$$

else

\{if (framerate $<10$ )

$$
\text { framerate }=\text { frame }+1 ;\}\}
$$

The threshold is bounded between 150 and 800 . If the threshold is equal to 800 and packet loss rate is still high, the framerate will be reduced to low down the packet loss rate. The transmitter adjusts $P S S$ and $P L L$ according to statistical data from receiver. Based on the practical Internet experiment, the network condition can be represented by sampling data each 10 seconds.

\section{EXPERIMENTAL RESULTS}

The adaptive network control scheme is tested on the 120 seconds salesman sequence. The spatial resolution is $320 \times 240$ for 8-bits gray level. The Internet transmission experiments are carried out between the video5 workstation and the cicsun06 workstation as shown in Table. 1. and in Fig. 7.

As shown in Fig. 8., the different statistical time is used to understand network condition by applying the adaptive control scheme. When the 10 seconds are chosen as the statistical time, the packet loss rate is the lowest.
Table. 2. shows the comparison of frame loss rate for various scheme. Testing time is at 08:00am during periods of weak day use. Table. 3. represents the frame loss rate during network peak day use. Because the network control scheme can adjust coding strategy according network condition, the frame loss rate is lower than source/channel coding in [1]. In comparison with the source/channel coding in [1], this adaptive network control scheme is presented to reduce $50 \%$ frame loss rate during the Internet weak day use condition and 58\% frame loss rate during the Internet peak day use condition.

\section{CONCLUSIONS}

A novel adaptive network control scheme for region-based hybrid coding algorithm is proposed in the above sections. The first order Markov model is used to control the network behavior. It is to meet the bandwidth of the network by minimizing data rates of transmitter. From the results of the real Internet experiments, this adaptive network control scheme is superior to the source/ channel coding.

\section{REFERENCE}

[1]. J. C. Bolot et al., "Adaptive Error Control for Packet Video in the Internet," Proceeding of IEEE Internal Conference on Image Processing, 1996.

[2]. Y. Chen etal., "A robust Coding Scheme for Packet Video," IEEE trans. on Commun., Vol. 40, No. 9, pp. 1491-1501, 1992.

[3]. H. T. Chen, P. C. Wu, Y. K. Lai, and L. G. Chen, "A multimedia Video Conference System: Using Region-based Hybrid Coding," IEEE trans. on Consumer Electronics, Vol. 42, No. 3, pp. 781-786, 1996. 


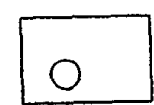

(a) Framaxn-1)

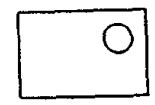

(b) Frame(a)
Figure I. Example of region base hybrid coding.

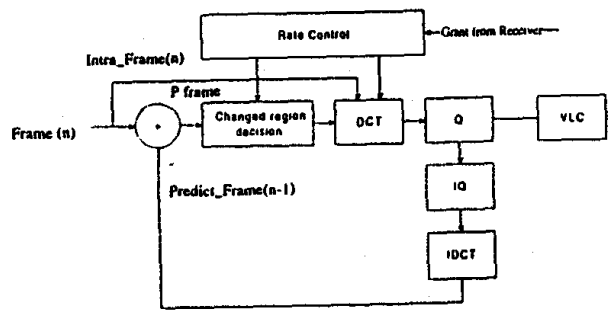

Figure 2. 8lock diagram of region base hybrid couling sysiem.

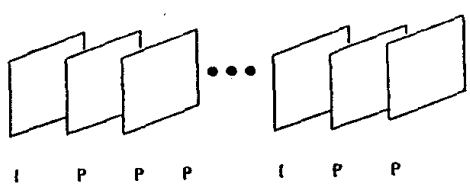

Figure 3. Frame type

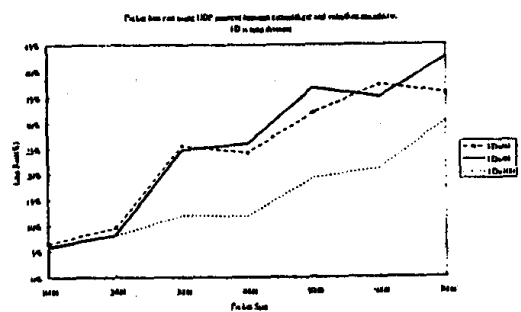

Figure 4. Packet loss ende for various packet size

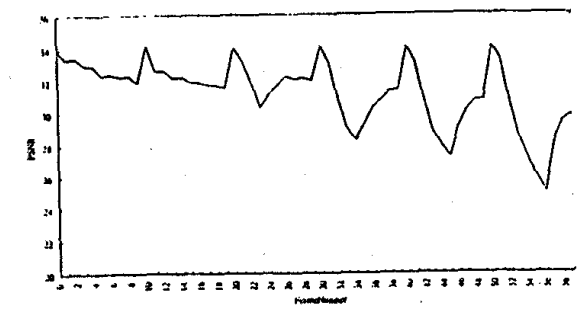

Figure 5. Frame loss simulation result of Region-base hybrid coding (Threshold=600). There are two successive frame loss at frame number 22 and 23. There are thece successive frame toss at frame number 32, 33 and 34. There are four successive irame loss al frame number $42,43,44$, and 45 . There are live successive frame loss at frame number $52,53,54,53$, and 56 .

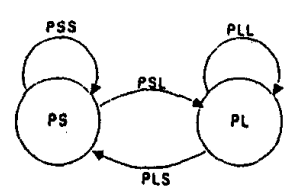

Figure 6. The state diagram of packet transmission in the network.

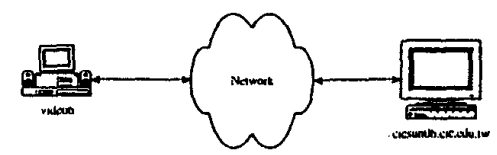

Figure 7. Testing environment

Table 1. Host computers used in our experiment

\begin{tabular}{|c|c|c|}
\hline hosiname + domainnume & P Address & Function \\
\hline videos.ec.ntu.edu.tw & 140.112 .20 .225 & receiver \\
\hline video.ee.ntu.edu.tw & 140.112 .18 .134 & rouler \\
\hline \multirow[t]{2}{*}{ nituee-router.ee.nitu.edu.tw } & 140.112 .18 .254 & router \\
\hline & 140.112 .41 .254 & rouler \\
\hline \multirow[t]{3}{*}{ nitu-rouler.ntu.edu.lw } & 140.112 .1 .254 & router \\
\hline & 134.208 .227 .111 & roultet \\
\hline & 134.208 .225 .110 & router \\
\hline NCHC-FDDI-NCTU.edu.tw & 134.208 .150 .113 & roviter \\
\hline NCTU-TI-CIC.nctu.edu,,$w$ & 140.126 .245 .253 & router \\
\hline \multirow[t]{2}{*}{ mbox-gw.cic.edu.sw } & 140.126 .24 .31 & roulter \\
\hline & 140.126 .26 .2 & rouler \\
\hline cicsun06.cic.edu.tw & 140.126 .25 .6 & transmitter \\
\hline
\end{tabular}

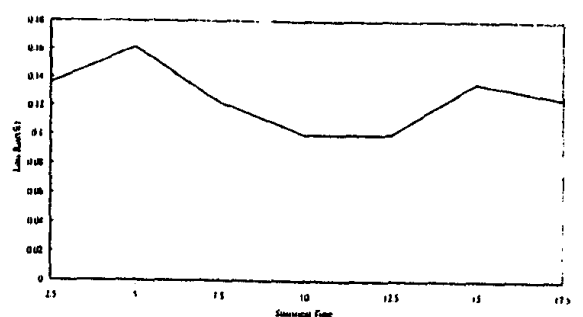

Figure 8. Statistical Time(sec) vs Loss Ratio (cpsl $=0.1 ; \mathrm{cpll}=0.1$ ).

Table 2. The frame loss comparison for various scheme. Network is in weak use. $($ cpsl $=0.1:$ cpll $=0.2)$

\begin{tabular}{|l|l|l|l|}
\hline Mechanism & Proposed & No nel work coatrol & Source/Channel Cuding III \\
\hline
\end{tabular} \begin{tabular}{|l|l|l|l|}
\hline Frame loss rate & $3 \%$ & $5 \%$ & $6 \%$ \\
\hline
\end{tabular}

Tabie 3. The irame loss comparison for various scheme. Network is in peak use. $($ cpsI $=0.15 ;$ cpll $=0.2$ )

\begin{tabular}{|l|l|l|l|}
\hline Mechanism & Proposed & No network control & Source/Channel Coding(II) \\
\hline
\end{tabular} \begin{tabular}{|l|l|l|l}
\hline Frame los rate & $8.9 \%$ & $16.67 \%$ & $21 \%$ \\
\hline
\end{tabular} 